\title{
A Need-Based Distributed Coordination Strategy for EV Storages in a Commercial Hybrid AC/DC Microgrid with an Improved Interlinking Converter Control Topology
}

\author{
Md Shamiur Rahman, Student Member, IEEE, M. J. Hossain, Senior Member, IEEE, \\ Junwei Lu, Senior Member, IEEE, H. R. Pota, Senior Member, IEEE,
}

\begin{abstract}
This paper presents a need-based distributed coordination strategy (NDCS) for multiple electric vehicle (EV) storages in an islanded commercial hybrid alternating-current (AC)/direct-current (DC) microgrid. The control capacity of the interlinking converter is enhanced by incorporating combined power-droop and voltage-droop strategies to leverage the coupling of $\mathrm{AC}$ and $\mathrm{DC}$ voltages. Therefore, the $\mathrm{AC}$ bus voltage can be regulated simultaneously by regulating only the DC bus voltage without affecting the power-sharing capabilities of the converter. The NDCS is proposed to coordinate the EV storages to regulate the DC bus voltage. The main objective of the NDCS is to decide whether the coordination of the available EV storages is to be done in a decentralised or a distributed manner. The mathematical model and the algorithm to deploy NDCS are developed to realise its application to a real system. The effectiveness of the control system is verified in a commercial hybrid AC/DC microgrid comprising one photovoltaic (PV) unit and four EV storages directly connected to the DC bus via DC/DC converters and four distributed-generation (DG) units connected to the AC bus using the conventional droop-control scheme. The performance of both controllers is tested under variable irradiation, commercial loading and various fault conditions. The results of the case studies demonstrate the efficacy of the overall system in terms of robustness for a variable generation-demand scenario, time delay, EV plug-and-play and fault conditions.
\end{abstract}

Index Terms-Hybrid AC/DC microgrid, interlinking converter, electric vehicles, distributed cooperative control, inverters.

\section{NOMENCLATURE}

$V_{D C}$

$V_{A C}^{R M S}$

$L_{f}, L_{c}, L_{G}$

$R_{f}, R_{c}, R_{G}$
Measured DC bus voltage.

Measured root-mean-square (RMS) voltage of the AC bus, also known as the point of common coupling (PCC).

DC bus voltage reference.

Filter, coupling and grid inductances.

Filter, coupling and grid resistances.

Md Shamiur Rahman and M. J. Hossain are with the School of Engineering, Macquarie University, NSW 2109, Australia (e-mail: mdshamiur.rahman@students.mq.edu.au; jahangir.hossain@mq.edu.au).

Junwei $\mathrm{Lu}$ is with the Griffith School of Engineering, Griffith University, Gold Coast, QLD 4222, Australia (e-mail: j.lu@griffith.edu.au).

H. R. Pota is with the School of Engineering and Information Technology, The University of New South Wales, Canberra, A.C.T. 2600, Australia (e-mail: h.pota@adfa.edu.au).

$r, r_{d}$
$C_{f}, C_{D C}$
$v_{o}^{a b c}, i_{o}^{a b c}$
$i_{l}^{a b c}$
$v_{o d}, i_{o d}$
$v_{o q}, i_{o q}$
$i_{l d}, i_{l q}$
$P$
${ }^{a b}$
$\psi_{v}$
$b_{v}$
$a_{i \times j}$
$D_{P}, D_{Q}$
$k_{e}$
$v_{B a t t}^{n}, i_{B a t t}^{n}$
$m$
$d_{\text {matt }}^{n}$

DC-side coupling and damping resistances.

Filter and DC side capacitances. Inverter output voltage and current in $a b c$ frame.

Inverter current before filter in natural $a b c$ frame.

$d$-axis inverter output voltage and current.

$q$-axis inverter output voltage and current.

$d-$ and $q-$ axis inverter output currents before filter.

Filtered active power.

Filtered reactive power.

Angular frequency.

Angle.

Feed-forward gain.

Droop coefficients of active and reactive power controller.

Droop coefficient of voltage-based droop controllers.

Voltage compensation factor for inverter interconnection loss.

Duty cycle of $n^{t h} \mathrm{EV}$ storage.

Modulation index of the inverter pulse-width-modulation (PWM) controller.

The largest eigen value of a particular matrix.

Reference values for corresponding variables.

Measured voltage and current of $n^{\text {th }} \mathrm{EV}$ before their corresponding DC/DC converter.

Cooperative-control input for voltage control loop.

Weight of the communication link from unit $j$ to $i$.

Coupling gain of the cooperative voltage control loop.

Acknowledgement signal for the voltage cooperation. 
$K_{p(.)}, K_{i(.)}, K_{d(.)} \quad$ Proportional-integral-derivative (PID) gains of interlinking inverter. $P, V$ and $I$ within parentheses represent control loops for power, voltage and current respectively.

$K_{p(.)}^{D C}, K_{i(.)}^{D C}$ PI gains of EV storage DC/DC converters.

$P_{A C(\text { load })}^{T o t}, Q_{A C(\text { load })}^{T o t} \quad$ Total active and reactive power drawn from the AC bus by the ACtype loads.

$P_{D C(\text { load })}^{T o t}$ $P_{A C(g e n)}^{T o t}, Q_{A C(g e n)}^{T o t}$ $P_{D C(\text { gen })}^{T o t}$ Total active power drawn from the DC bus by the DC-type loads.

Total active and reactive power generations by AC-type DG units.

Total active power generation by DC-type DG units.

\section{INTRODUCTION}

W ITH a view to leveraging the advantages of both alternating current $(\mathrm{AC})$ and direct current (DC) types of power systems, recently a hybrid AC/DC microgrid structure is proposed in [1]-[2]. This particular structure is gaining momentum due to its several advantages including minimum AC/DC conversion loss, higher efficiency, less effort in voltage synchronisation and feasibility of source-load connection [3][4]. However, the control structure of a hybrid microgrid is more complex due to the coexistence of multiple buses of different electric natures. Supplementary complexities are introduced when dynamic energy-storage systems (ESS) like electric vehicles (EVs) are plugged in on a massive scale, either to charge themselves or to provide ancillary vehicleto-grid (V2G) services, such as active and reactive power support, voltage and frequency regulation, valley filling, peakload shaving etc. [5]-[6]. Consequently, the hybrid AC/DC microgrid is unlikely to be implemented in residential areas in the near future, because of higher uncertainties and complexities [7]. Thus, suitable applications for a hybrid AC/DC microgrid structure could be commercial or industrial vicinities [8]-[9]. Moreover, commercial buildings are using nearly $32 \%$ of the overall energy generation, and at the same time these buildings are responsible for emitting almost $30 \%$ of the $\mathrm{CO}_{2}$ related to energy consumption. Sustainable sources like photovoltaic (PV) units, fuel cells and small-scale wind turbines can be incorporated to reduce this emission. The efficiency of the overall system can be further improved by utilising the power generated from the EVs parked in a commercial vicinity [10][11]. However, additional research attention is required for the effective utilisation and control of EVs in a commercial-grade microgrid.

Microgrids require a definite control structure due to the presence of power electronic converters and non-inertial sources as well as their ability to work in both islanded and grid-tied modes. The concept of a hierarchical control structure for both conventional power systems and for all types of microgrids (i.e. AC, DC and hybrid AC/DC) is well-accepted in the literature [12]. This structure involves a hierarchy of primary, secondary and tertiary layers of control. Of those layers the primary control layer is well researched and various types of decentralized droop controls are predominantly studied and used for this layer. The main objectives of both the primary and secondary control layers are to ensure voltage and frequency stability by compensating for respective deviations, whereas the tertiary control layer is more focused on optimal power flow and economic dispatch operation in the grid-tied mode. Both secondary and tertiary control layers can achieve their individual objectives by adopting either a centralized (for small-scale microgrids) or a distributed approach (commercialgrade microgrids or interconnected microgrids) [12]-[13]. However, the centralized approach has some limitations such as single-point failures, extensive computational burden for the processing units and expensive communication networks. As a result, distributed control approaches are gaining attention particularly for the secondary and tertiary control layers. This control structure requires sparse communication among neighboring distributed-generation (DG) units and utilises local parameters, such as voltage, frequency and power requirements to regulate them, which in turns reduces the computational burden of the processing units and reduces the overall cost with improved reliability [14]-[15].

Recently application of distributed control in microgrids has been a topic of interest due to the above-mentioned advantages over centralized control structures. For instance, these controllers are developed for AC microgrids [16]-[17] to achieve proportional power sharing and voltage regulation. The controller has been applied to DC microgrids [18] and is later extended for multiple DC microgrid clusters [19]-[20]. The performance of the developed controller is later improved to consider the impacts of communication latency [21]-[22], uncertain communication topology [23], communication link failure and parameter uncertainties [15]. Consensus-based distributed control structures for ESS are developed in some recent literature [24]-[25] to achieve certain objectives, such as state-of-charge (SOC) balancing and energy-level balancing. An SOC-based distributed cooperative controller is developed to reduce distribution feeder-voltage swelling due to high PV penetration [26]-[27]. A distributed consensus algorithm is utilised for PV-battery based single-phase islanded microgrids [28]. However, none of these analyses considered simultaneous $\mathrm{AC}$ and DC bus voltage regulation, which is a primary requirement, particularly for an islanded hybrid AC/DC microgrid. Moreover, effective utilisation of EV storage in a microgrid through proper coordination requires additional research.

Several distributed control schemes are proposed to coordinate ESSs considering them as active cooperative agents. However, most of these literature consider the controllers used, either in the primary or the secondary layer to be fully distributed in nature. Distributed controllers are indeed attractive options to coordinate multiple storages; nevertheless, problems the use of communication and the extent of computational burden persist. As a result, an efficient algorithm is required, which will ensure the period and the scenario when the distributed controllers need to be activated. Motivated by these challenges, this paper presents a need-based distributed coordination strategy (NDCS) for the V2G-capable EV storages connected to the DC bus of the microgrid to regulate 
the DC bus voltage of the hybrid AC/DC microgrid. Firstly, the correlation between the AC-bus RMS voltage and the DC bus voltage is established using a voltage-based droop control method [29]-[30]. The conventional interlinking converter (IC) control structure is extended with combined power- and voltage-droop control schemes. As a result, one type of voltage regulation ensures regulation of other types of voltage without affecting the power-sharing capabilities of the converters. The main contribution of this paper can be summarised as follows:

1) An improved IC-control structure is proposed by unifying proportional-derivative (PD) power-based and voltage-based droop control structures. This improved control topology ensures better transient performance and provides coupling between the AC-bus RMS and the DC bus voltage without affecting the power-sharing capability of the converter. This control topology is costeffective as it substantially utilises the apparent-power (kVA) ratings of the converter without overloading it.

2) An integrated EV storage controller is proposed which can work in both decentralised and distributed manner. This controller ensures DC-bus voltage regulation, which results in zero circulating currents. As a result, the efficiency and the lifetime of the EV storages are improved. Additionally, this controller interacts with the IC to aid in regulating the $\mathrm{AC}$ bus voltage. As a result, simultaneous $\mathrm{AC}$ and $\mathrm{DC}$ bus voltage regulation is achieved.

3) A novel strategy named NDCS is proposed that takes the EV availability into account to activate need-based distributed coordination of multiple EVs. By utilising this algorithm, the advantages of both decentralised and distributed controllers can be obtained.

4) Unlike existing distributed controllers, the proposed need-based controller exhibits robust fault-tolerance while operating in pair with the improved IC-control structure, which ensures reliable control framework.

5) Interaction between the EV storage controllers and the IC controller affects the dynamic response of the hybrid microgrid. Moreover, there are substantial effects of time delay on the performance of the IC when the EV storages are controlled in a distributed manner. Therefore, a dynamic mathematical model for the IC controller is derived considering the interaction and the delay effects.

The remainder of the paper is organized as follows. Section II presents the proposed IC control topology and its mathematical model. The general formulation of the proposed NDCS for the EV storages is presented in Section III. In Section IV, case studies involving a commercial hybrid AC/DC microgrid with four EV storages in various scenarios are presented to demonstrate the controller performance. Finally, concluding remarks are presented in Section V.

\section{Proposed IC CONTROL TOPOLOGY}

The IC acts as an interface between the AC and DC subgrids while the microgrid enters into a hybrid AC/DC microgrid operational mode. The IC controller has three inner control loops namely power controller, voltage controller and current controller as shown in Fig. 1. The dynamic modeling of these control loops is briefly described in the following subsections.

\section{A. Power-based droop control}

The instantaneous voltage $v_{o}^{a b c}$ and current $i_{o}^{a b c}$ after the LC filter and the inverter output current $v_{l}^{a b c}$ are measured and transformed through the Park transformation. The real and reactive power calculations are carried out as follows [16], [31]:

$$
\begin{aligned}
p & =\frac{3}{2}\left(v_{o d} i_{o d}+v_{o q} i_{o q}\right) \\
q & =\frac{3}{2}\left(v_{o q} i_{o d}-v_{o d} i_{o q}\right)
\end{aligned}
$$

The fundamental components of $p$ and $q$ are extracted using a low-pass filter (LPF) with a cutoff frequency $\omega_{c}$. The filtered active and reactive power are as follows:

$$
\begin{aligned}
& P=\frac{\omega_{c}}{s+\omega_{c}} p \\
& Q=\frac{\omega_{c}}{s+\omega_{c}} q
\end{aligned}
$$

Voltage and frequency are regulated via droop control during islanded microgrid operation. The usual $X / R$ ratio of a microgrid is very high due to the high inductance of the inductors present in LC filters, particularly for a commercialgrade microgrid. As a result, the well-known $P / f$ and $Q / V$ droop control can be applied to regulate the frequency and the voltage of the system as follows [12]:

$$
\begin{aligned}
\omega & =\omega^{*}-D_{P}\left(P-P^{*}\right) \\
V & =V^{*}-D_{Q}\left(Q-Q^{*}\right)
\end{aligned}
$$

It should be noted that the designed controller can also be applied to microgrids with a low $X / R$ ratio using the inverse droop equations. The dynamic performance of the conventional droop controllers is poor and can be improved by implementing PD-compensated droop control, which enables robust microgrid mode transitions and improved fault tolerance capabilities [32]. Now, assuming the $d$-axis to be aligned with the line voltage measured by the phase-locked-loop (PLL), we get $v_{o q}^{*}=0$. Therefore, $V \approx v_{o d}^{*}$. Then the droop equations become:

$$
\begin{aligned}
\omega & =\omega^{*}-K_{p(P)} D_{P}\left(P-P^{*}\right)-K_{d(P)} D_{P} \frac{d}{d t}\left(P-P^{*}\right) \\
V=v_{o d}^{*} & =V^{*}-K_{p(P)} D_{Q}\left(Q-Q^{*}\right)-K_{d(P)} D_{Q} \frac{d}{d t}\left(Q-Q^{*}\right)
\end{aligned}
$$

$\omega^{*}$ is a global parameter, and a constant value can be assigned to it. For instance, for the system frequency of $50 \mathrm{~Hz}$, $\omega^{*}=314.16 \mathrm{rad} / \mathrm{s}$. Conventionally $V^{*}, P^{*}$ and $Q^{*}$ are taken as constants as well. However, for this paper all of these three parameters are adaptive. This method ensures robustness in dynamic load changing, uninterrupted active/reactive powersharing capabilities of the converter, and simultaneous AC and DC bus voltage regulation. The following subsection will discuss how $P^{*}, Q^{*}$ and $V^{*}$ are dynamically generated. 


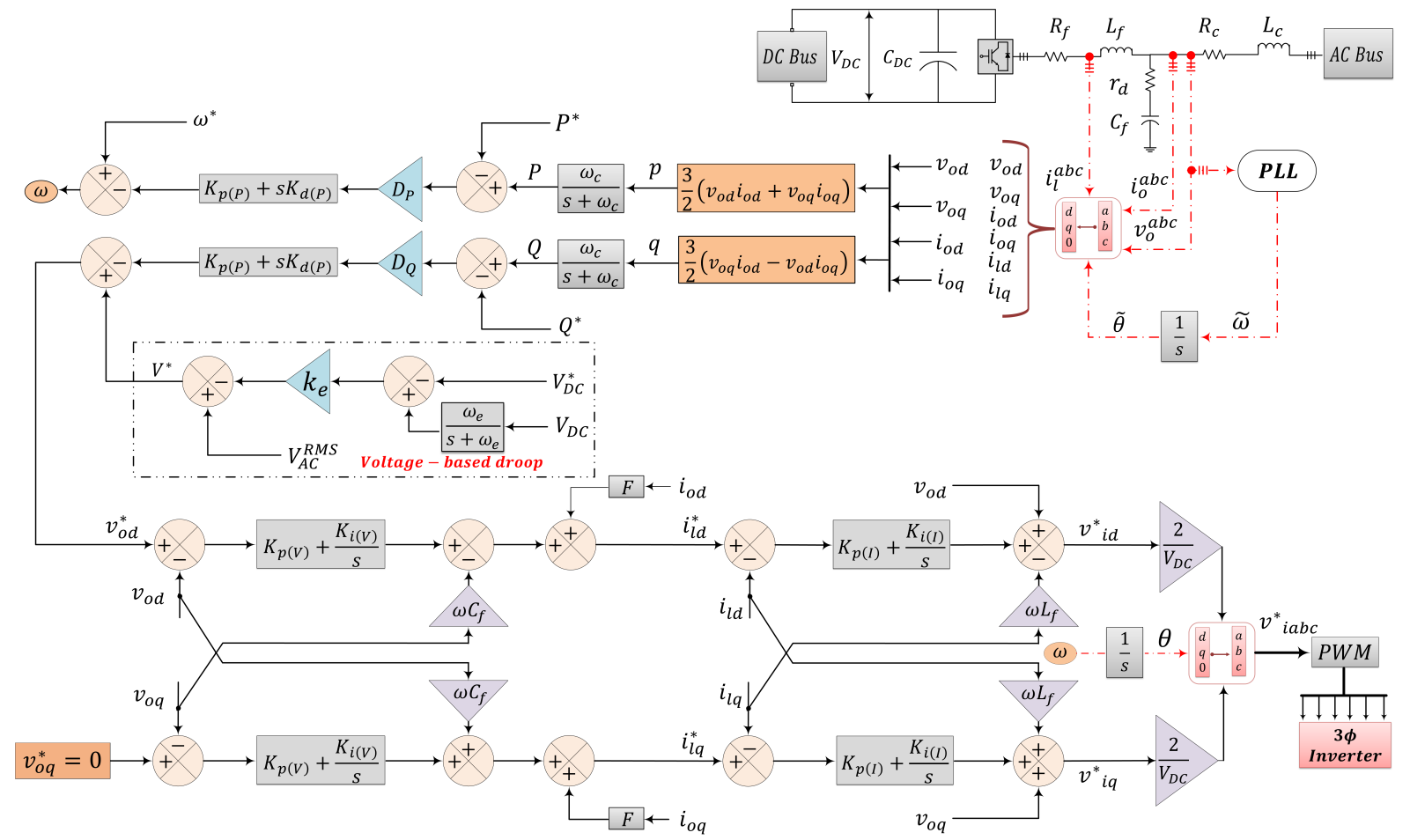

Fig. 1: IC control structure with voltage-based droop.

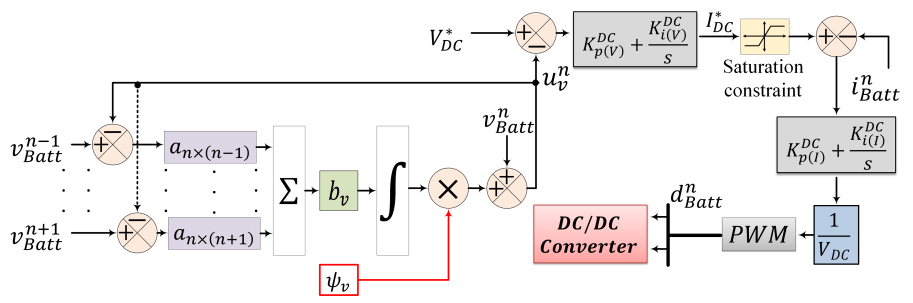

Fig. 2: EV converter controller with distributed cooperative control loop.

\section{B. Generation of $P^{*}, Q^{*}$ and $V^{*}$}

The references for the active power $\left(P^{*}\right)$, reactive power $\left(Q^{*}\right)$ and the AC-side voltage $\left(V^{*}\right)$ need to be adaptive, so that the IC controller performs robustly in changing network dynamics. Let us define three variables $(\mathcal{A}, \mathcal{B}, \mathcal{C}) \subseteq \mathbf{R}$ as follows:

$$
\begin{aligned}
& \mathcal{A}=P_{A C(\text { load })}^{T o t}-P_{A C(\text { gen })}^{T o t} \\
& \mathcal{B}=Q_{A C(\text { load })}^{T o t}-Q_{A C(\text { gen })}^{T o t} \\
& \mathcal{C}=P_{D C(\text { load })}^{T o t}-P_{D C(\text { gen })}^{T o t}
\end{aligned}
$$

Line losses should be added, if known, to all of (5)-(7). However, line losses are not considered in this paper. The normal operation of a hybrid $\mathrm{AC} / \mathrm{DC}$ microgrid refers to zero energy tranfer between the AC and DC subgrids. In order to preserve the KVA ratings of the converters, the normal operation requires $\sqrt{\mathcal{A}^{2}+\mathcal{B}^{2}}=\mathcal{C}$; however, deviations might occur due to overloading or surplus energy generation. These deviations will lead to hybrid AC/DC microgrid operation. Then $P^{*}$ and $Q^{*}$ for the hybrid AC/DC microgrid operation are to be defined as follows:

$$
\begin{aligned}
& P^{*}=\left\{\begin{array}{lll}
0 & \text { for } & \sqrt{\mathcal{A}^{2}+\mathcal{B}^{2}}=\mathcal{C} \\
\mathcal{A} & \text { for } & \sqrt{\mathcal{A}^{2}+\mathcal{B}^{2}}>\mathcal{C} \\
\mathcal{C} & \text { for } & \sqrt{\mathcal{A}^{2}+\mathcal{B}^{2}}<\mathcal{C}
\end{array}\right. \\
& Q^{*}=\left\{\begin{array}{lll}
0 & \text { for } & \sqrt{\mathcal{A}^{2}+\mathcal{B}^{2}}=\mathcal{C} \\
\mathcal{B} & \text { for } & \sqrt{\mathcal{A}^{2}+\mathcal{B}^{2}}>\mathcal{C} \\
0 & \text { for } & \sqrt{\mathcal{A}^{2}+\mathcal{B}^{2}}<\mathcal{C}
\end{array}\right.
\end{aligned}
$$

The inverter output AC voltage is proportionally related to the DC link voltage. The relation is presented in [33] and later elaborated in [8]. Vandoorn et al. in [29]-[30] utilise this relationship of voltages of different electric natures to control the inverters of DG units. This relation can be expressed as

$$
V_{D C} \approx \underbrace{2 \times\left(\frac{\Lambda}{m}\right) \times\left(\frac{\sqrt{2}}{\sqrt{3}}\right)}_{\alpha} \times V_{L-L}^{A C}
$$

where $V_{L-L}^{A C}=$ peak-to-peak magnitude of the phase-to-phase voltage of the $\mathrm{AC}$ bus and $\alpha=$ slope of the linear relation presented in (10).

Then the $V^{A C} / V^{D C}$ droop equation presented in [29]-[30] can be expressed as

$$
V^{*}=V_{A C}^{R M S}+K_{e}\left(V_{D C}-V_{D C}^{*}\right)
$$

Implementation of the control scheme in (11), combined with the power-based droop in the power control loop of the IC controller gives the opportunity, particularly for the islanded microgrid scenario, to achieve simultaneous quasi-decoupled power sharing and AC and DC bus-voltage regulation. Representing the delay using an LPF with the cutoff frequency of $\omega_{e}$ where $\omega_{e}>\omega_{c}$, the proposed IC control topology is 
illustrated in Fig. 1. It can be seen from Fig. 1 that if the DC bus voltage is tightly regulated (i.e. $V_{D C}-V_{D C}^{*} \approx 0$ ) then the whole IC control structure operates under the conventional strategy. As a result, the power sharing remains unaffected. Herein, by utilising the information obtained from (8) - (9); and by the perturbing (2), (4) and (11) the state-space dynamic model of the proposed power-control loop of the IC controller can be obtained as follows:

$$
\begin{aligned}
& {\left[\begin{array}{c}
Q-Q^{*} \\
P-P^{*} \\
V^{*}
\end{array}\right]=A\left[\begin{array}{c}
Q-Q^{*} \\
P-P^{*} \\
V^{*}
\end{array}\right]+B\left[\begin{array}{c}
q-Q \\
p-P \\
V_{D C}-V_{D C}^{*} \\
V_{A C}^{R M} \\
\omega^{*}
\end{array}\right]} \\
& {\left[\begin{array}{c}
v_{o d}^{*} \\
\omega
\end{array}\right]=C\left[\begin{array}{c}
Q-Q^{*} \\
P-P * \\
V^{*}
\end{array}\right]+D\left[\begin{array}{c}
q-Q \\
p-P \\
V_{D C}-V_{D C}^{*} \\
V_{A C}^{R M} \\
\omega^{*}
\end{array}\right]} \\
& A=\left[\begin{array}{ccc}
0 & 0 & 0 \\
0 & 0 & 0 \\
0 & 0 & -\omega_{e}
\end{array}\right] ; B=\left[\begin{array}{ccccc}
\omega_{c} & 0 & 0 & 0 & 0 \\
0 & \omega_{c} & 0 & 0 & 0 \\
0 & 0 & K_{e} \omega_{e} & \omega_{e} & 0
\end{array}\right] \text {; } \\
& C=\left[\begin{array}{ccc}
-K_{p(P)} D_{Q} & 0 & 1 \\
0 & -K_{p(P)} D_{P} & 0
\end{array}\right] \text {; } \\
& D=\left[\begin{array}{ccccc}
-K_{d(P)} D_{Q} \omega_{c} & 0 & 0 & 0 & 0 \\
0 & -K_{d(P)} D_{P} \omega_{c} & 0 & 0 & 1
\end{array}\right]
\end{aligned}
$$

It is evident from the $A$ matrix of the proposed power control loop that the system is only dependent upon the time delay attributed to the distributed controllers of EVs. It is also clear that $\operatorname{eig}(A)<0$ for all values of $\omega_{e}$. As a result, the system is inherently stable for acceptable time delays. However, the controller, operating in the distributed mode can reach critical stability for unlikely large time delays in range of seconds.

\section{Voltage and current controllers}

Voltage and current controllers are developed utilising decoupled d-q axis PI controllers with feed-forward gains [16], [24]. The combined dynamic equations for both voltage and current controllers can be obtained as follows:

$$
\begin{aligned}
\dot{\phi}_{o d} & =v_{o d}^{*}-v_{o d} \\
\dot{\phi}_{o q} & =v_{o q}^{*}-v_{o q} \\
\dot{\lambda}_{l d} & =i_{l d}^{*}-i_{l d} \\
\dot{\lambda}_{l q} & =i_{l q}^{*}-i_{l q} \\
\imath_{l d}^{*} & =F i_{o d}-\omega C_{f} v_{o q}+K_{p(V)} \dot{\phi_{o d}}+K_{i(V)} \phi_{o d} \\
\imath_{l q}^{*} & =F i_{o q}-\omega C_{f} v_{o d}+K_{p(V)} \dot{\phi_{o q}}+K_{i(V)} \phi_{o q} \\
v_{i d}^{*} & =v_{o d}-\omega L_{f} i_{l q}+K_{p(I)} \dot{\lambda_{l d}}+K_{i(I)} \lambda_{l d} \\
v_{l q}^{*} & =v_{o q}+\omega L_{f} i_{l d}+K_{p(I)} \dot{\lambda_{l q}}+K_{i(I)} \lambda_{l q}
\end{aligned}
$$

where $\dot{\phi}_{o d}$ and $\dot{\phi}_{o q}$ are state variables corresponding to the PI controller of the voltage controller whereas $\dot{\lambda}_{l d}$ and $\dot{\lambda}_{l q}$ are state variables corresponding to the PI controller of the current controller.

\section{NEED-BASED DistribUted COORDINATION STRATEGY}

Let us consider a microgrid of $N$ number of EV storages communicating with each other through a sparse communication network. The communication graph, also known as a weighted directed graph (digraph), representing the interconnection of nodes can be denoted by $\mathcal{G}=\left(\mathcal{V}_{\mathcal{G}}, \mathcal{E}_{\mathcal{G}}, \mathcal{A}_{\mathcal{G}}\right)$, where $\mathcal{V}_{\mathcal{G}}=\left\{\mathcal{V}_{1}, \mathcal{V}_{2}, \mathcal{V}_{3}, \ldots . \mathcal{V}_{n}\right\}$ is the set of all nodes, $\mathcal{E}_{\mathcal{G}} \subset \mathcal{V}_{\mathcal{G}} \times \mathcal{V}_{\mathcal{G}}$ is the set of pairs of nodes also known as edges, $\mathcal{A}_{\mathcal{G}}=\left[a_{i j}\right]_{N \times N}$ is the weighted adjacency matrix of dimension $N \times N$ that gives information regarding the interconnectivity of nodes. The communication between node $i$ and node $j$ can be presented as follows:

$$
a_{i \times j}=\left\{\begin{array}{cc}
1 & \text { if } \\
0 & \text { otherwise }
\end{array} \quad(i, j) \in \mathcal{E}_{\mathcal{G}}\right.
$$

The in-degree matrix is $D_{G}^{i n}=\operatorname{diag}\left\{d_{i}^{i n}\right\}$, i.e. the diagonal matrix of $d_{i}^{i n}=\sum_{j=0}^{n} a_{i j}$. Similarly the out-degree matrix is $D_{G}^{\text {out }}=\operatorname{diag}\left\{d_{i}^{\text {out }}\right\}$, i.e. the diagonal matrix of $d_{i}^{\text {out }}=\sum_{i=0}^{n} a_{j i}$. For an undirected communication network with bidirectional information flow, $D_{G}^{o u t}=D_{G}^{i n}$. As a result, the Laplacian matrix $\mathcal{L}_{\mathcal{G}}$, defined as $\mathcal{L}_{\mathcal{G}}=D_{G}^{i n}-\mathcal{A}_{\mathcal{G}}$, is considered balanced [34].

All EV storages are designed with the distributed dynamic average consensus protocols shown in Fig. 2. This protocol uses local information of the $n^{t h} \mathrm{EV}$ storage and the information measured from its neighbors to converge to an average output voltage $u_{v}^{n}$, given by:

$$
\begin{aligned}
u_{v}^{n}(t) & =v_{\text {Batt }}^{n}(t)+\underbrace{\psi_{v} b_{v} \int_{0}^{t} \sum_{j \in N} a_{n \times j}\left(v_{b a t t}^{j}(\tau)-u_{v}^{n}(\tau)\right) d \tau}_{\Delta v} \\
& =v_{\text {Batt }}^{n}(t)+\Delta v
\end{aligned}
$$

Following this protocol, the output voltages of the EV storages for any step changes will converge at an average value of the neighboring units at $t \rightarrow \infty$, as long as there exists a spanning tree and the communication delay $\left(T_{d}\right)$ satisfies the condition [35]

$$
T_{d} \leq \frac{\pi}{2 \lambda_{\max }\left(\mathcal{L}_{\mathcal{G}}\right)}
$$

The nominal value of the DC bus voltage is fixed for a particular application. As a result, without loss of generality it can be considered that all the EV storages connected to the DC bus have access to this reference value. This enables the entire controller to act in a leaderless manner. Fig. 3 illustrates the NDCS for cooperative V2G-capable EV storages. Each $\mathrm{EV}$ storage collects the terminal voltage information of its neighboring units $n-1$ and $n+1$. The DC bus voltage deviation, $\Delta V_{D C}$ is checked for the acceptable operating range, i.e. $-3 \%-+5 \%$ [8]. If $\Delta V_{D C}$ is within the acceptable operating range then zero is assigned for $\psi_{v}$. This results in $\Delta v=0$ such that $u_{v}^{n}=v_{\text {Batt }}^{n}$. As a result, the EV storage controllers start operating in the decentralized mode. On the other hand, when $\Delta V_{D C}$ exceeds the acceptable range, then a substantial number of EV storages need to work cooperatively to regulate the DC bus voltage. As a result, when this situation 
occurs, NDCS will check the availability of V2G-capable EV storages. The availability constraints can be set as follows:

Storage-capacity constraints for the $n$th EV can be set as $\left(S O C_{n}^{\text {max }}-S O C_{n}^{\text {initial }}\right) Q_{n}^{\text {Rated }} \leq \Delta Q_{n}^{\Delta T_{n}} \leq\left(S O C_{n}^{\text {initial }}\right.$ $\left.S O C_{n}^{\text {min }}\right) Q_{n}^{\text {Rated; }}$; where, $S O C_{n}^{\max }, S O C_{n}^{\text {min }}, S O C_{n}^{\text {initial }}$ are the maximum, minimum and the initial state-of-charge (SOC) of the $n$th EV storage respectively; $Q_{n}^{\text {Rated }}$ and $\Delta Q_{n}^{\Delta T_{n}}$ are the rated capacity and the capacity for the duration of $\mathrm{V} 2 \mathrm{G}$ operation $\left(\Delta T_{n}\right)$ of the $n$th EV storage respectively.

The converter capacity for the $n$th EV storage can be depicted as $-P_{n}^{\max } \leq P_{n}^{E V} \leq P_{n}^{\max }$ and $-Q_{n}^{\max } \leq Q_{n}^{E V} \leq$ $Q_{n}^{\max }$;

where $P_{n}^{\max }$ and $Q_{n}^{\max }$ are the maximum allowable active and reactive power respectively and $P_{n}^{E V}$ and $Q_{n}^{E V}$ are the output active and reactive powers respectively for the $n$th $\mathrm{EV}$ storage. However, the reactive power capacity will not be a concern when EVs are operating through DC/DC converters.

A flexible user experience can be preserved by setting a constraint such as $S O C_{n}^{\text {final }} \geq S O C_{n}^{\text {pre-set}}$; where $S O C_{n}^{\text {final }}$ and $S O C_{n}^{\text {pre-set }}$ are the SOC of the $n$th EV storage after the V2G participation and the user-defined pre-set SOC respectively.

Now if all the constraints are satisfied then $\psi_{v}=1$ will be assigned which enables the EV storage controllers to operate in the distributed mode. However, if the constraints are not satisfied then the NDCS will keep the EV storage controller in the decentralised mode by preserving $\psi_{v}=0$. Later on, it will initiate non-critical load curtailment to keep $\Delta V_{D C}$ within the limit. The grid reconnection command will be initiated for the microgrid as the last resort of regulation. For both decentralised and distributed modes of operation, $u_{v}^{n}$ is updated according to (15) and $I_{D C}^{*}$ is generated using (17), which results in the generation of $d_{\text {Batt }}^{n}$ for the DC/DC converters of the EV storages using (18). In the final step the output voltage, $v_{\text {Batt }}^{n}$ of the EV storage is broadcast to its neighboring units $n-1$ and $n+1$.

$$
\begin{gathered}
I_{D C}^{*}=\left(V_{D C}^{*}-u_{v}^{n}\right)\left(K_{p(V)}^{D C}+\frac{K_{i(V)}^{D C}}{s}\right) \\
d_{\text {Batt }}^{n}=\frac{\left(I_{D C}^{*}-i_{B a t t}^{n}\right)\left(K_{p(I)}^{D C}+\frac{K_{i(I)}^{D C}}{s}\right)}{V_{D C}}
\end{gathered}
$$

Storage lives can be enhanced by preserving pre-defined SOC levels for all EVs. As a result, it is important to maintain that level when EVs are operating in the decentralised mode. According to [36], the SOC of a battery is a function of time and output current of the battery i.e. $S O C_{n}=f\left(t, i_{\text {Batt }}^{n}\right)$. It can be observed from (17) and (18) that $i_{B a t t}^{n}=f\left(I_{D C}^{*}\right)$. It implies that, by limiting the dynamic value of $I_{D C}^{*}$, the SOC level can be maintained during the decentralised mode. Assuming that each EV has the maximum power capacity of $20 \mathrm{~kW}$ with the output reference DC bus voltage $520 \mathrm{~V}$, in this paper we have assumed the maximum and minimum values of $I_{D C}^{*}$ to be $\pm 40 \mathrm{~A}$.

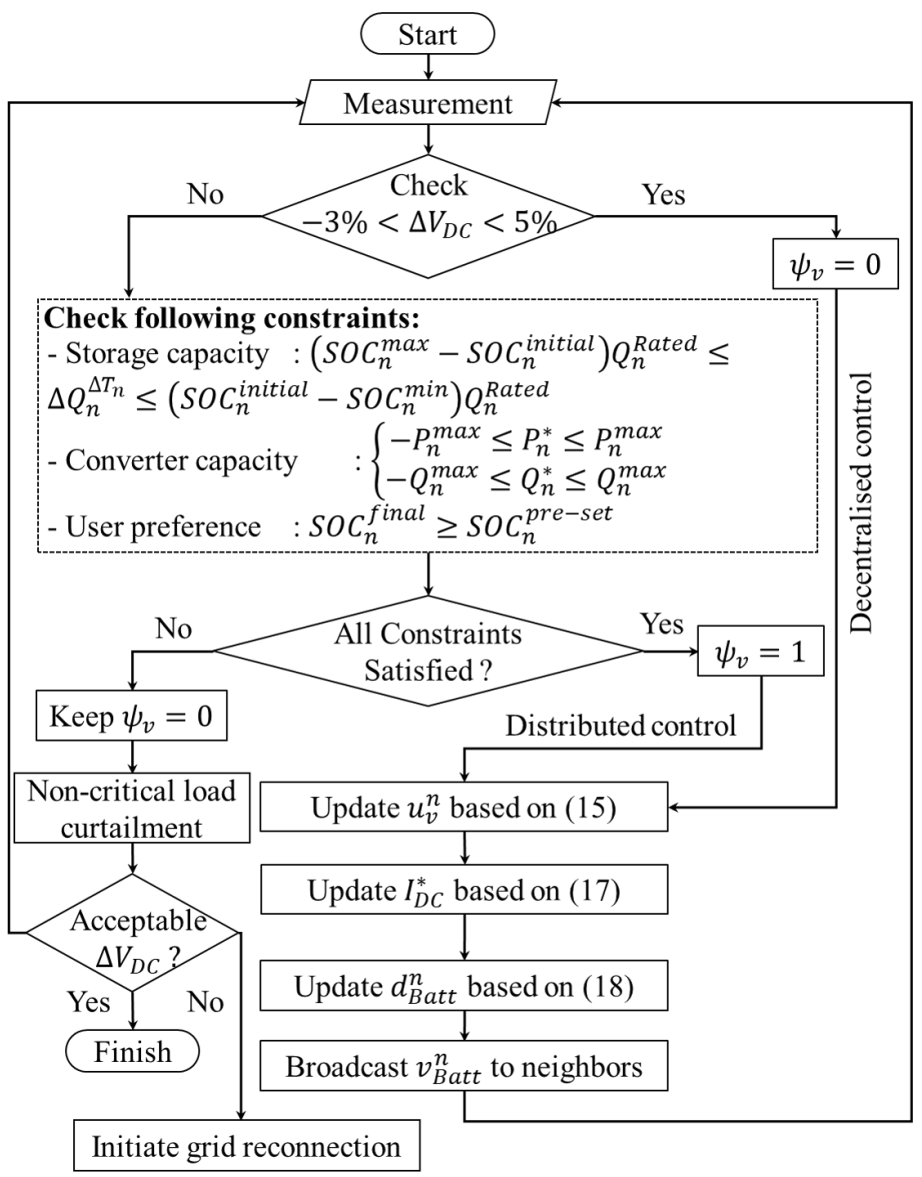

Fig. 3: Need-based distributed coordination strategy of EV storages.

\section{CAse studies}

Several case studies are carried out to demonstrate the performance of the proposed controller. For all the case studies, the microgrid is considered to be islanded. Case A is carried out considering islanded operation, with step load changes to verify the performance of the proposed NDCS. Concurrent variable PV generation and real commercial loading are considered for case B to demonstrate the efficacy of the controller under intermittent supply and demand. This case study also demonstrates the efficacy of the proposed controller in the distributed mode with the conventional one presented in [2]. In case $\mathrm{C}$, the effects of time delay on the controller performance are demonstrated. The impacts of user-preferred disconnection is explored in case D to demonstrate plug-andplay features. Fault-robustness of the controller is assessed in case E with three-phase symmetric and DC short circuit fault scenarios.

The designed hybrid AC/DC microgrid model is an extended version of the university-based commercial hybrid AC/DC microgrid as presented in [8]. A simplified single-line diagram of the model is illustrated in Fig. 4. The AC subgrid consists of four DG units controlled by the conventional droopcontrol scheme [13] without any inter-unit communication. All DG units are connected to the AC bus through respective LCL filters. The DC subgrid is designed with four EV storages and one PV unit. The EV storages are connected to the DC 


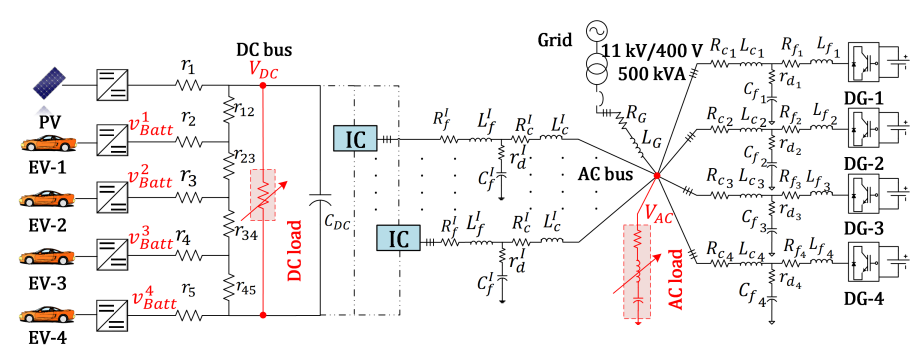

Fig. 4: Single-line diagram of the test hybrid AC/DC microgrid.

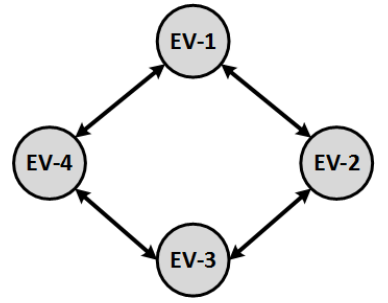

Fig. 5: Communication graph of EVs.

bus through DC fast chargers which are controlled by the developed distributed cooperative controllers. The bidirectional connectivity graph among the four EVs is shown in Fig. 5. The DC/DC converter of the PV unit is controlled by a maximum-power-point tracking (MPPT) controller. The DC bus is interfaced with the AC bus through the IC. DC loads are connected at the DC bus terminal and AC loads are connected at the AC bus terminal. The load profile as shown in Fig. 9 and the solar irradiation data used for the case studies of this paper are obtained using OSI PI software that uses a centralized communication structure. The system parameters and ratings of all units are given in Table I. Additional information regarding the hardware setups, communication protocols used for data collection and monitoring along with building schematics can be obtained from [8].

\section{A. Case A: Step load change}

The objective of this case study was to compare the performance of NDCS with the decentralised coordination of EVs under sudden load change. From $t=0 \mathrm{~s}$ to $4 \mathrm{~s}$ EVs were operating a under the decentralised mode. During this period, the DC bus was loaded with $40 \mathrm{~kW}$ DC load. All EV

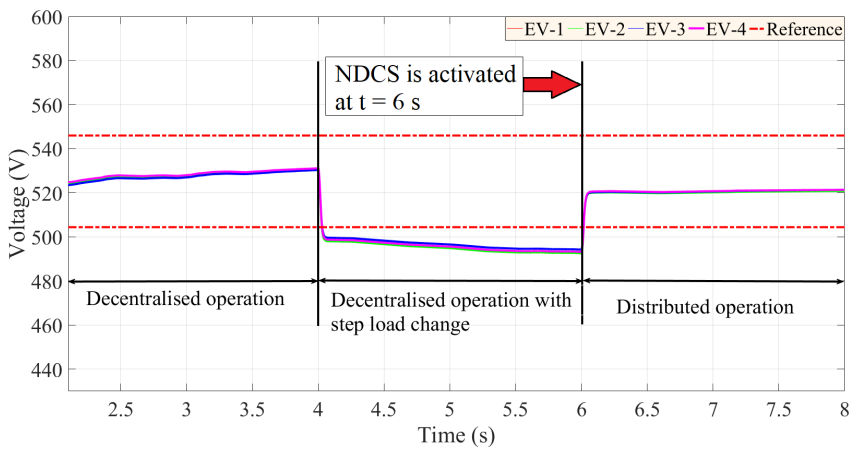

Fig. 6: $v_{\text {Batt }}^{1}, v_{\text {Batt }}^{2}, v_{\text {Batt }}^{3}$ and $v_{\text {Batt }}^{4}$ for case A.
TABLE I: System parameters

\begin{tabular}{|c|c|c|c|}
\hline \multicolumn{4}{|c|}{ Energy nodes } \\
\hline $\mathrm{PV}$ & $30 \mathrm{~kW}$ & & \\
\hline EV- $(1,2,3,4)$ & $\begin{array}{l}24,41, \\
30 \text { and } 23 \mathrm{kWh}\end{array}$ & $\begin{array}{l}\text { Initial EV battery } \\
\text { voltages, }\left(v_{\text {Batt }}^{1},\right. \\
v_{\text {Batt }}^{2}, v_{\text {Batt }}^{3} \\
\left.\text { and } v_{B a t t}^{4}\right)\end{array}$ & $\begin{array}{l}465,580,500 \\
\text { and } 520 \mathrm{~V}\end{array}$ \\
\hline DG-( $(1,2,3,4)$ & $17 \mathrm{kVA}$ & & \\
\hline \multicolumn{4}{|c|}{ Line parameters } \\
\hline Nominal $V_{A C}^{R M S}$ & $240 \mathrm{~V}$ & $\overline{L_{f}}$ & $10 \times 10^{-3} \mathrm{H}$ \\
\hline$V_{D C}^{*}$ & $520 \mathrm{~V}$ & $C_{f}$ & $250 \times 10^{-6} \mathrm{~F}$ \\
\hline Frequency & $50 \mathrm{~Hz}$ & $R_{c}, R_{G}$ & $0.03 \Omega$ \\
\hline$C_{D C}$ & $150 \times 10^{-4} \mathrm{~F}$ & $L_{c}, L_{G}$ & $3.5 \times 10^{-4} \mathrm{H}$ \\
\hline$R_{f}$ & $2 \times 10^{-3} \Omega$ & $r_{d}$ & $0.65 \Omega$ \\
\hline $\begin{array}{l}r_{1}, r_{2} \\
r_{3}, r_{4}\end{array}$ & $0.02 \Omega$ & $\begin{array}{l}r_{12}, r_{23} \\
r_{34}, r_{45}\end{array}$ & $0.05 \Omega$ \\
\hline \multicolumn{4}{|c|}{ Control parameters } \\
\hline Power controller & & Voltage controlle & \\
\hline$\omega_{c}, \omega_{e}$ & $31.42,314.16 \mathrm{rad} / \mathrm{s}$ & $\mathrm{F}$ & 0.19 \\
\hline$\omega^{*}$ & $314.16 \mathrm{rad} / \mathrm{s}$ & $K_{p(V)}$ & 0.11 \\
\hline$D_{P}$ & $3.14 \times 10^{-4}$ & $K_{i(V)}$ & 2.2 \\
\hline$D_{Q}$ & $1.36 \times 10^{-3}$ & \multicolumn{2}{|l|}{ Current controller } \\
\hline$k_{e}$ & 1 & $K_{p(I)}$ & 0.16 \\
\hline$K_{p(P)}$ & $2 \times 10^{-5}$ & $K_{i(I)}$ & 200 \\
\hline$K_{d(P)}$ & $2 \times 10^{-7}$ & & \\
\hline \multicolumn{4}{|c|}{ EV storage controller } \\
\hline$K_{p(V)}^{D C}$ & 1.3 & $K_{p(I)}^{D C}$ & 0.03 \\
\hline$K_{i(V)}^{D C}$ & 6 & $K_{i(I)}^{D C}$ & 1.1 \\
\hline$b_{v}$ & 1.5 & & \\
\hline
\end{tabular}

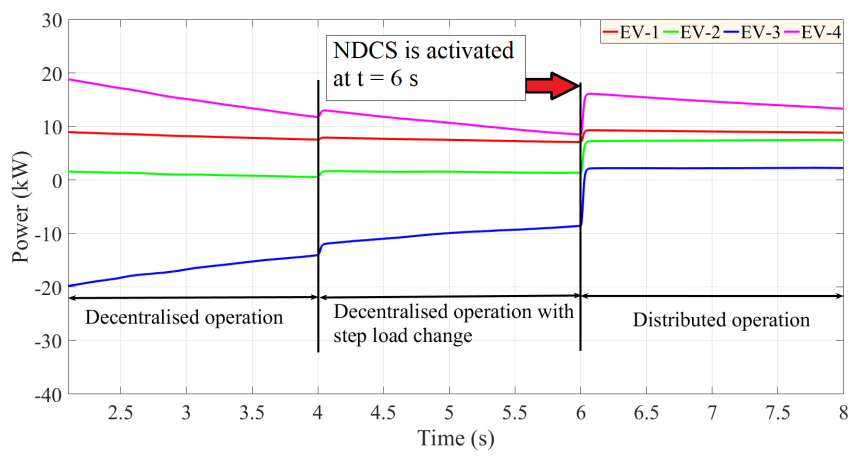

Fig. 7: Power supplied or consumed by the EVs for case A.

converters were designed to generate or consume a maximum of $20 \mathrm{~kW}$ power. As a result, the maximum and minimum saturation points for $I_{D C}^{*}$, as mentioned earlier, were chosen as $\pm 40 \mathrm{~A}$. Over utilisation of EV converters, particularly during decentralised operation, can be avoided by putting this limit in the current-control loops of EV converters. At $t=4 \mathrm{~s}$, a step load of $25 \mathrm{~kW}$ was switched on for the duration of

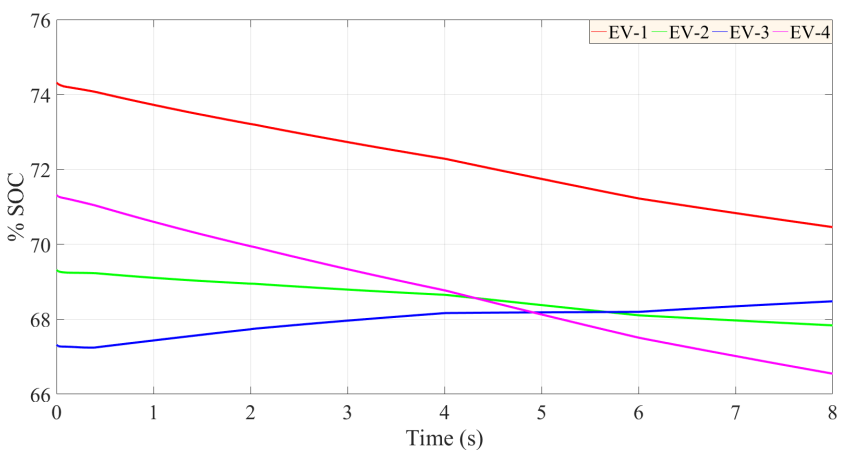

Fig. 8: \%SOC profile for all EVs for case A. 


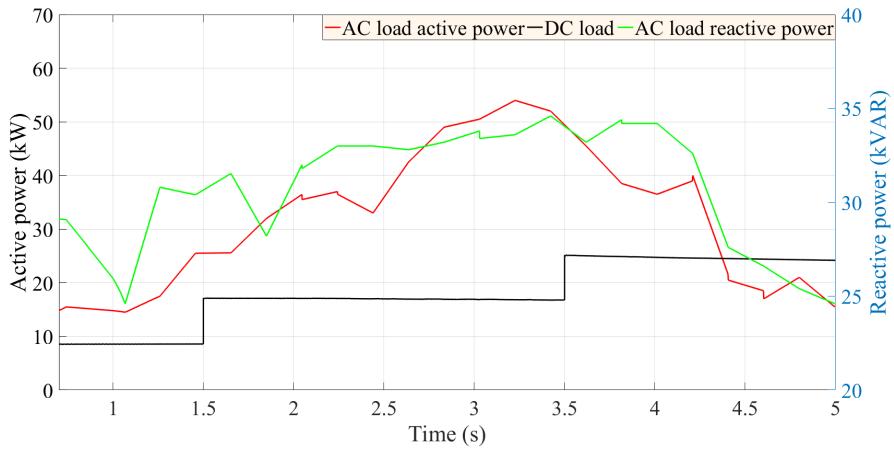

Fig. 9: Commercial AC and DC load profiles

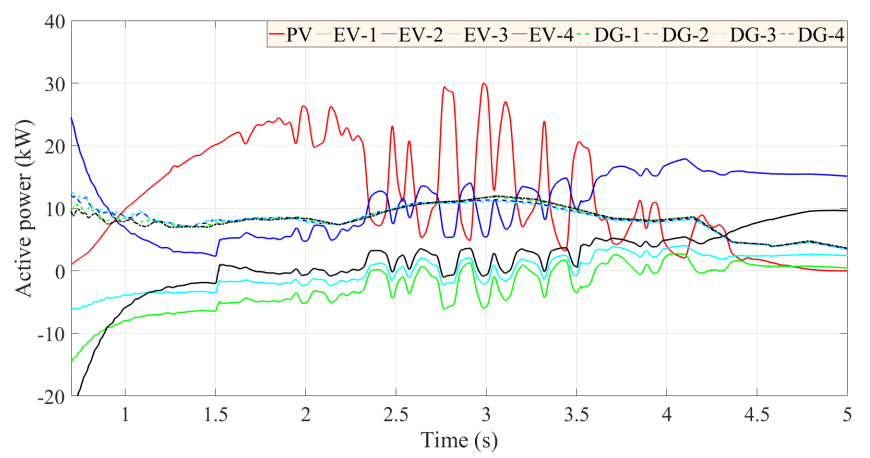

Fig. 10: Varying output power of all DG units. DC type DG units are shown in solid lines and the AC type DG units are shown in dotted lines.

2 s. As a result, the DC bus voltage dropped down below the lower reference level as shown in Fig. 6. The standard acceptable voltage ranges for the $\mathrm{AC}$ and $\mathrm{DC}$ bus voltages are shown in dotted red lines. The standard references for the AC bus voltage is in between 0.94 per-unit $(\mathrm{pu})$ to $1.1 \mathrm{pu}$ [24]. For the DC bus voltage, this range is in between $-3 \%-+5 \%$ of the nominal voltage [8]. In order to restore the DC bus voltage to its reference value, at $t=6 \mathrm{~s}$ NDCS was activated. The objective of the NDCS was to provide regulation-up service by ensuring that all EVs generate additional power by discharging. The generated or consumed power of all EVs and their corresponding \%SOC are shown in Fig. 7 and Fig. 8 respectively. As can be seen there, initially EV-1, EV-2 and EV-4 were in the discharging mode, whereas EV-3 was in the charging mode. When the NDCS was activated, all EVs including EV-3 checked their individual constraints and started discharging. This results in a restored DC bus voltage. Apart from the voltage restoration, it can be observed from Fig. 6 that NDCS demonstrates more stable DC bus voltage regulation capability than the decentralised operation.

\section{B. Case B: Variable PV generation and commercial loading}

For case $\mathrm{B}$, the islanded hybrid AC/DC microgrid was exposed to variable PV generation due to fluctuating irradiation. The main aim of this case study was to verify the performance of the designed controller under variable PV generation, as its output is random and variable in nature. The DC bus and AC bus were loaded with commercial load profiles as shown in Fig. 9. Both conditions (i.e. variable PV generation and load profile) were considered simultaneously to accurately represent a realistic scenario. The NDCS for the EV storages was activated at $t=0 \mathrm{~s}$. The communication links among neighbors were considered ideal without time delay. The output active powers of all the DG units are shown in Fig. 10. The output powers of all the DG units at the AC side were alike because of their fixed droop coefficients. The output voltages of the EV storage converters and the AC bus RMS voltages are shown respectively in Figs. 11a and 11b. It can be observed from Fig. 11a that, despite the differences in the initial terminal voltages, all of the EV storages eventually converged to the desired voltage level. Likewise, due to the coupling of the DC bus voltage with the AC bus RMS voltage, the convergence of the DC side voltage assists in controlling the AC bus RMS voltage to be within the acceptable range.

During islanded microgrid condition, it is important that all operating ICs should have four-quadrant operational capability. This means that the converter can transfer both active and reactive power in between two subgrids. As a result, the inverter will have four modes of operation (rectifier, inverter, capacitive and inductive modes). During the rectifier mode, the inverter will transfer power to the DC subgrid from the AC subgrid, whereas, during the inverter mode it will transfer power to the AC subgrid from the DC subgrid. The normalised DC bus voltage for all the cases are shown in Fig. 11g. The DC bus voltage for this case is shown in red in Fig. 11g. It can be seen that the voltage is properly regulated through the proposed control scheme. The tracking performance of the proposed controller for the reactive power reference is shown in Fig. 11d in comparison with the conventional decentralised control scheme presented in [2]. It can be observed that the proposed controller provides superior reference-tracking performance without any steady-state error.

\section{Case C: Effects of time delay}

EV storage controllers need to withstand an obvious latency while operating under the distributed mode, which requires sparse communication among neighbors. The main purpose of this case study is to evaluate the controller performance under a communication time delay. It should be noted that the usual communication delays for WiFi-based networks are typically $20 \mathrm{~ms}$ [37]. However, for this case study a $30 \mathrm{~ms}$ time delay was assumed and the effects on the controller performance were observed. NDCS were considered active at $t=0 \mathrm{~s}$. The AC bus RMS voltage for this case is shown in blue color in Fig. 11b. Fig. 11c presents the output voltages of the EV storages for this case study. The output voltages of all EVs took approximately $1.5 \mathrm{~s}$ to converge when the time delay is $30 \mathrm{~ms}$. It can be observed from Fig. 11c that due to the $30 \mathrm{~ms}$ delay there were slight magnitude deviations in the beginning for the voltages of the EV storages, which also impacted the AC bus voltage as shown in Fig. 11b. Nevertheless, the $\mathrm{AC}$ bus voltage remained in acceptable range as shown in Fig. 11b. The DC bus voltage also maintained the acceptable range for this case as presented in Fig. $11 \mathrm{~g}$ in blue color. It should be noted that the performance of the controller in terms of convergence speed can be further improved by increasing the level of connectivity. However, 

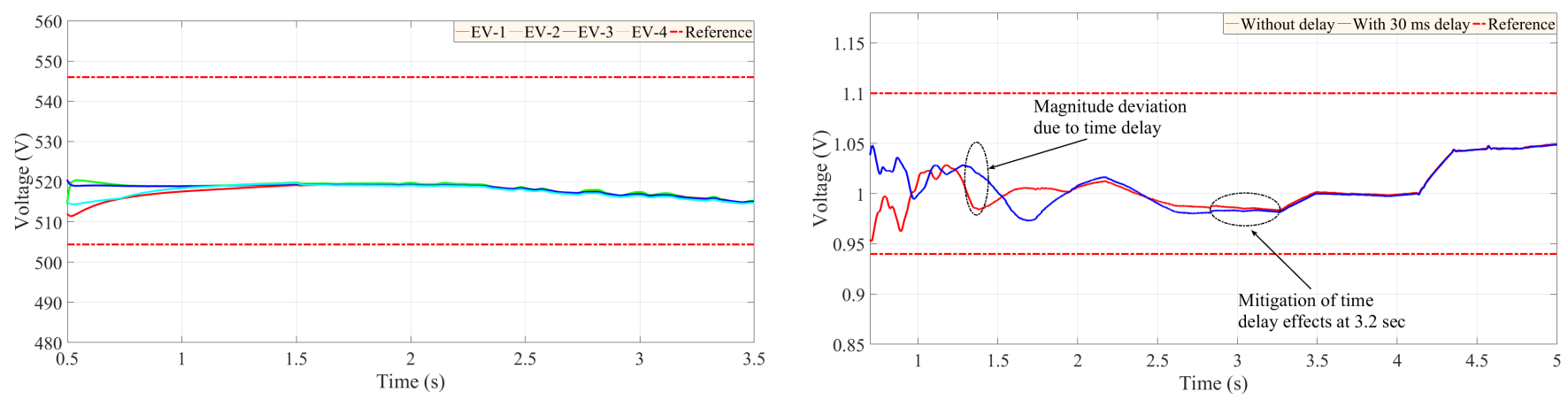

(a) $v_{\text {Batt }}^{1}, v_{\text {Batt }}^{2}, v_{\text {Batt }}^{3}$ and $v_{\text {Batt }}^{4}$ for case B.

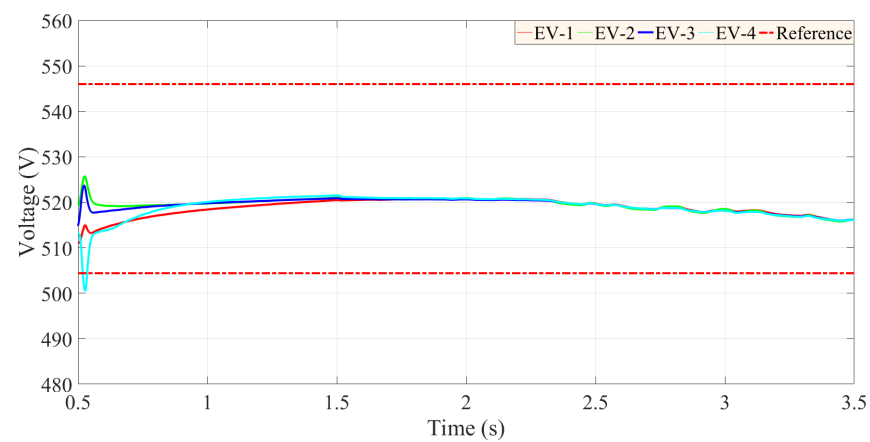

(b) AC-bus RMS voltage case B (red) and case C (blue).

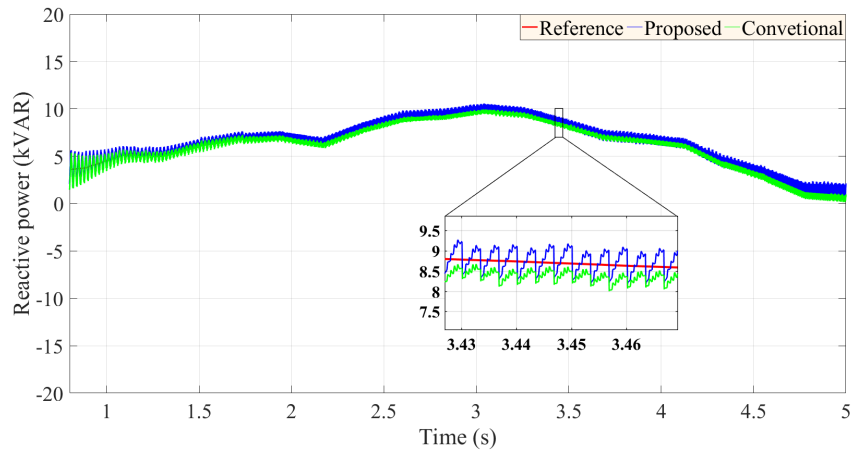

(c) $v_{\text {Batt }}^{1}, v_{\text {Batt }}^{2}, v_{\text {Batt }}^{3}$ and $v_{\text {Batt }}^{4}$ for case C.

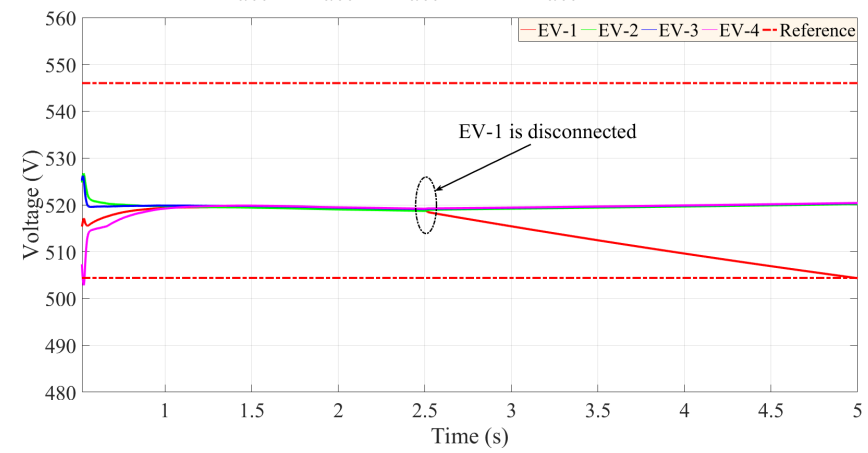

(d) Reference-tracking performance of the proposed controller.

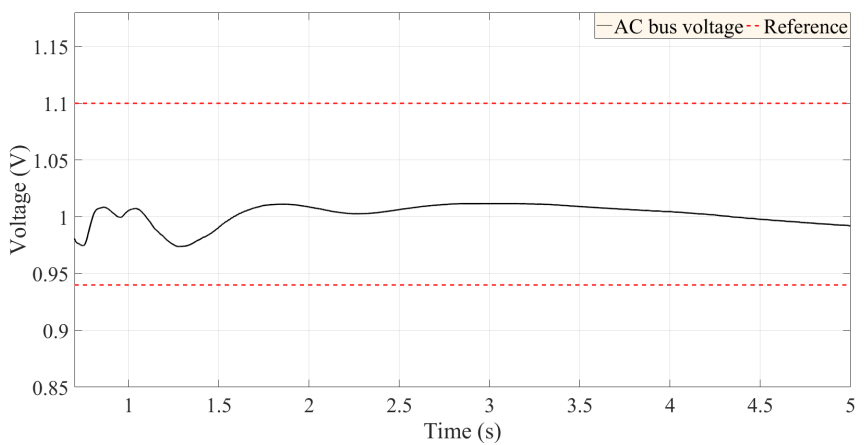

(e) $v_{\text {Batt }}^{1}, v_{\text {Batt }}^{2}, v_{\text {Batt }}^{3}$ and $v_{\text {Batt }}^{4}$ for case D.

(f) AC-bus RMS voltage for case D.

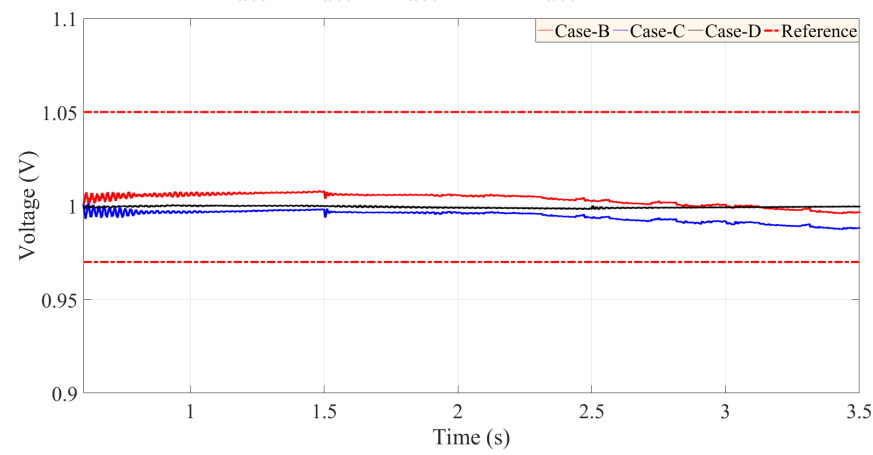

(g) Normalised DC bus voltage for cases B, C and D.

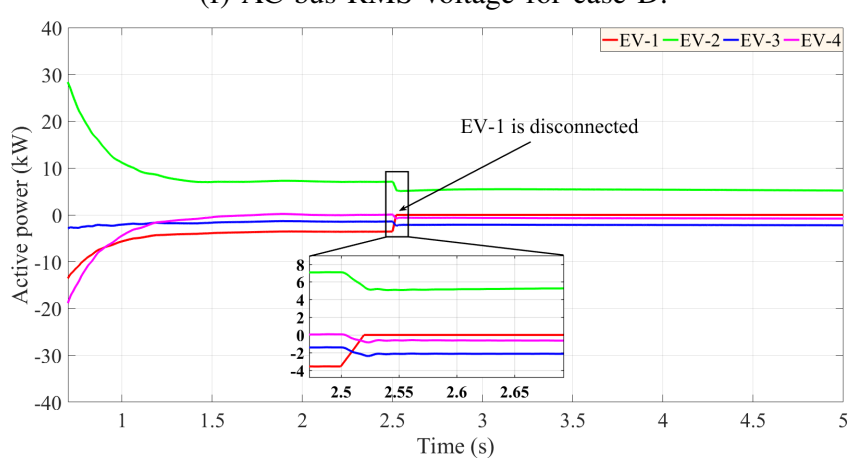

(h) Output power of EV storages for case D.

Fig. 11: Main results of cases B, C and D. 

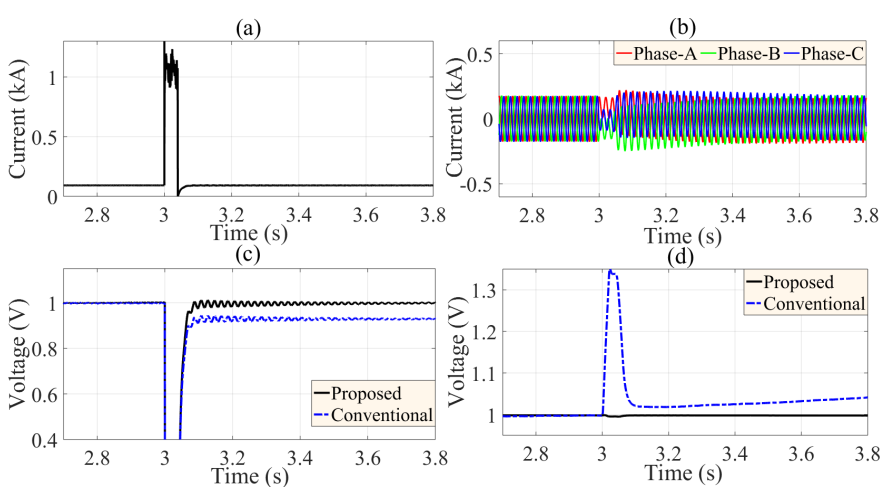

Fig. 12: Performance evaluation of the proposed scheme under the DC short circuit fault for case $\mathrm{E}$. The fault was applied at $t=3 \mathrm{~s}$ for the duration of 40 ms. (a) Load current at the DC bus. (b) Load current at the AC bus. (c) Normalised DC bus voltage with the proposed distributed controller (solid black) and with the conventional decentralised controller (dotted blue). (d) AC bus RMS voltage in per unit (pu) with the proposed distributed controller (solid black) and with conventional decentralised controller (dotted blue).

this will increase the communication-based cost [35]. Overall, it is evident from the result that both the IC controller and the distributed cooperative controller are robust to time-delay effects.

\section{Case D: User-preferred disconnection}

The objective of this case study is to evaluate the controller performance when participating EV users disconnect the V2G service without sending any notification signal. The controller should work in a dynamic condition where EV owners will have the flexibility to include or exclude their EVs for V2G operations. For this case study, both types of loads were kept constant. For the AC load a fixed $70 \mathrm{~kW}+j 30$ kvar was used and for the DC load a fixed $30 \mathrm{~kW}$ was used.

The NDCS was activated at the beginning of the simulation. At $\mathrm{t}=2.5 \mathrm{~s}$, user of EV-1 decided to disconnect the V2G service while EV-2, EV-3 and EV-4 was active under the NDCS. The output voltages of EV storages maintain the required voltage level as shown in Fig. 11e to regulate the DC-bus voltage as shown in black in Fig. 11g. As the DC bus voltage is properly regulated, it keeps the AC-bus RMS voltage within the acceptable limit as presented in Fig. 11f. The output powers of the EVs for this case study are shown in Fig. 11h. It can be observed that the output power of EV-1 becomes zero when disconnected. However, the load, EV-3 and EV-4, is supported partially by the high-capacity EV storage EV-2. The rest of the power is exported from the AC subgrid for this particular case.

\section{E. Case E: Performance evaluation under faults}

The robustness of the proposed distributed controller was evaluated under two types of fault scenario. At first, a short circuit fault was applied at the DC bus at $t=3 \mathrm{~s}$ for the duration of $30 \mathrm{~ms}$. During that period, the DC bus was loaded with $55 \mathrm{~kW}$ and the AC bus was loaded with $200 \mathrm{~kW}+j 70$ kvar. The load current at the DC bus experienced a spike due to the fault condition as shown in Fig. 12a. Due to the fault at the DC terminal, the load current at the AC bus was temporarily
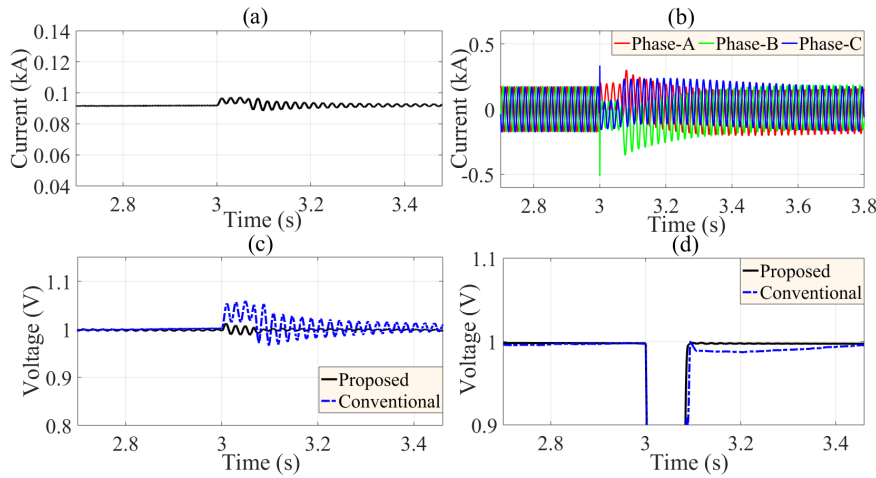

Fig. 13: Performance evaluation of the proposed scheme under three-phase AC symmetric fault for case $\mathrm{E}$. The fault was applied at $t=3 \mathrm{~s}$ for the duration $60 \mathrm{~ms}$ (a) Load current at the DC bus. (b) Load current at the AC bus. (c) Normalised DC bus voltage with the proposed distributed controller (solid black) and with the conventional decentralised controller (dotted blue). (d) AC bus RMS voltage in per unit (pu) with the proposed distributed controller (solid black) and with conventional decentralised controller (dotted blue).

affected as presented in Fig. 12b. Figs. 12c and 12d represent the DC bus voltage and the AC bus RMS voltage respectively for this case study. Voltages are shown with the solid black line for the proposed distributed controller and with the dotted blue line for the conventional decentralised controller. As compared to the conventional decentralised controller, the proposed distributed controller effectively performs post-fault voltage regulation for both $\mathrm{AC}$ and $\mathrm{DC}$ bus voltages with zero steady state error.

The performance of the proposed controller was further validated under three-phase symmetric fault applied at the AC bus. The fault was applied at $t=3 \mathrm{~s}$ for the period of 60 ms (3 cycles for a $50 \mathrm{~Hz}$ system). The loading conditions for both buses were kept the same. The load current at the DC bus and the AC bus are shown in Figs. 13a and 13b. The DC and the AC bus RMS voltages for this case study are shown in Figs. 13c and 13d. Both voltages are marked with solid black and dotted blue lines respectively for the proposed distributed controller and the conventional decentralised controller. It is evident from Figs. 13c and 13d that both bus voltages are effectively regulated when the fault is cleared. The conventional decentralised controller exhibited oscillatory performance for the DC bus voltage and higher settling time for the AC bus RMS voltage as compared to the proposed distributed controller.

\section{CONClusion}

This paper proposes a novel algorithm to coordinate EV storages in a commercial hybrid AC/DC microgrid. Based on the DC-bus voltage condition, the algorithm switches the coordination among multiple EV storages from decentralised to distributed mode or vice versa. Firstly, the conventional IC control topology is enhanced by using a combination of the PD-compensating power-droop and voltage-droop control schemes. This development ensures improved transient response along with the coupling between the AC-bus RMS voltage and the DC-bus voltage. This results in the capability of regulating one bus voltage by regulating the other. Secondly, a mode-changing EV storage controller is designed which 
can operate in decentralised or distributed mode. Finally, a new coordination strategy namely NDCS, is proposed that ensures when and how long the EV storages need to operate in distributed mode to regulate the DC bus voltage. The NDCS also takes into account the storage and converter capacities along with the user preferences for both decentralised and distributed operation. Case studies are carried out to demonstrate the performance of the developed controller under concurrent variable PV generation and commercial loading. The effects of time delay and user-preferred disconnection of operating EVs are explored through case studies. Furthermore, the robustness of the controller are quantified with the DCside short circuit fault and the three-phase AC symmetric fault. It is evident from the comparative analysis that the developed controller operated by NDCS can provide robust performance for voltage regulation and power sharing within a hybrid AC/DC microgrid.

\section{REFERENCES}

[1] X. Liu, P. Wang, and P. C. Loh, "A hybrid AC/DC microgrid and its coordination control," IEEE Trans. Smart Grid, vol. 2, no. 2, pp. 278 286, June 2011.

[2] P. C. Loh, D. Li, Y. K. Chai, and F. Blaabjerg, "Autonomous control of interlinking converter with energy storage in hybrid AC/DC microgrid," IEEE Trans. Ind. Appl., vol. 49, no. 3, pp. 1374-1382, May 2013.

[3] N. Eghtedarpour and E. Farjah, "Power control and management in a hybrid AC/DC microgrid," IEEE Trans. Smart Grid, vol. 5, no. 3, pp. 1494-1505, May 2014.

[4] P. Wang, L. Goel, X. Liu, and F. H. Choo, "Harmonizing AC and DC: A hybrid AC/DC future grid solution," IEEE Power Energy Mag., vol. 11, no. 3, pp. 76-83, May 2013.

[5] M. Yilmaz and P. T. Krein, "Review of the impact of vehicle-to-grid technologies on distribution systems and utility interfaces," IEEE Trans. Power Electron., vol. 28, no. 12, pp. 5673-5689, December 2013.

[6] M. S. Rahman, F. Rafi, M. J. Hossain, and J. Lu, "Power control and monitoring of the smart grid with EVs," Vehicle-to-Grid: Linking Electric Vehicles to the Smart Grid, p. 107, 2015.

[7] M. S. Rahman, M. J. Hossain, and J. Lu, "Utilization of parked ev-ess for power management in a grid-tied hybrid ac/dc microgrid," in 2015 Australasian Universities Power Engineering Conference (AUPEC), Sept 2015, pp. 1-6.

[8] — , "Coordinated control of three-phase AC and DC type EV-ESSs for efficient hybrid microgrid operations," Energy Conversion and Management, vol. 122, pp. 488 - 503, 2016.

[9] M. S. Rahman, M. J. Hossain, F. H. M. Rafi, and J. Lu, "EV charging in a commercial hybrid AC/DC microgrid: Configuration, control and impact analysis," in 2016 Australasian Universities Power Engineering Conference (AUPEC), September 2016, pp. 1-6.

[10] J. V. Roy, N. Leemput, F. Geth, J. Buscher, R. Salenbien, and J. Driesen, "Electric vehicle charging in an office building microgrid with distributed energy resources," IEEE Trans. Sustain. Energy, vol. 5, no. 4, pp. 1389-1396, October 2014.

[11] N. Mahmud and A. Zahedi, "Review of control strategies for voltage regulation of the smart distribution network with high penetration of renewable distributed generation," Renew. Sustain. Energy Rev, vol. 64, no. Supplement C, pp. 582 - 595, 2016.

[12] J. M. Guerrero, J. C. Vasquez, J. Matas, L. G. de Vicuna, and M. Castilla "Hierarchical control of droop-controlled AC and DC microgrids: A general approach toward standardization," IEEE Trans. Ind. Electron., vol. 58, no. 1, pp. 158-172, January 2011.

[13] J. M. Guerrero, P. C. Loh, T. L. Lee, and M. Chandorkar, "Advanced control architectures for intelligent microgrids - part II: Power quality, energy storage, and AC/DC microgrids," IEEE Trans. Ind. Electron., vol. 60, no. 4, pp. 1263-1270, April 2013.

[14] F. Dorfler, J. W. Simpson-Porco, and F. Bullo, "Breaking the hierarchy: Distributed control and economic optimality in microgrids," IEEE Trans. Control Netw. Syst., vol. 3, no. 3, pp. 241-253, September 2016.

[15] M. J. Hossain, M. A. Mahmud, F. Milano, S. Bacha, and A. Hably, "Design of robust distributed control for interconnected microgrids," IEEE Trans. Smart Grid, vol. PP, no. 99, pp. 1-12, 2015.
[16] A. Bidram, A. Davoudi, F. L. Lewis, and J. M. Guerrero, "Distributed cooperative secondary control of microgrids using feedback linearization," IEEE Trans. Power Syst., vol. 28, no. 3, pp. 3462-3470, August 2013.

[17] A. Bidram, F. L. Lewis, and A. Davoudi, "Distributed control systems for small-scale power networks: Using multiagent cooperative control theory," IEEE Control Syst. Mag., vol. 34, no. 6, pp. 56-77, December 2014.

[18] V. Nasirian, S. Moayedi, A. Davoudi, and F. L. Lewis, "Distributed cooperative control of DC microgrids," IEEE Trans. Power Electron., vol. 30, no. 4, pp. 2288-2303, April 2015.

[19] Q. Shafiee, T. Dragicevic, J. C. Vasquez, and J. M. Guerrero, "Hierarchical control for multiple DC-microgrids clusters," IEEE Trans. Energy Convers., vol. 29, no. 4, pp. 922-933, December 2014.

[20] S. Moayedi and A. Davoudi, "Distributed tertiary control of DC microgrid clusters," IEEE Trans. Power Electron., vol. 31, no. 2, pp. 17171733, February 2016.

[21] J. Lai, H. Zhou, X. Lu, X. Yu, and W. Hu, "Droop-based distributed cooperative control for microgrids with time-varying delays," IEEE Trans. Smart Grid, vol. 7, no. 4, pp. 1775-1789, July 2016.

[22] X. Lu, X. Yu, J. Lai, J. Guerrero, and H. Zhou, "Distributed secondary voltage and frequency control for islanded microgrids with uncertain communication links," IEEE Trans. Ind. Informat., vol. PP, no. 99, pp. $1-1,2016$

[23] W. Liu, W. Gu, W. Sheng, X. Meng, S. Xue, and M. Chen, "Pinningbased distributed cooperative control for autonomous microgrids under uncertain communication topologies," IEEE Trans. Power Syst., vol. 31, no. 2, pp. 1320-1329, March 2016.

[24] T. Morstyn, B. Hredzak, and V. G. Agelidis, "Distributed cooperative control of microgrid storage," IEEE Trans. Power Syst., vol. 30, no. 5, pp. 2780-2789, September 2015.

[25] T. Morstyn, A. Savkin, B. Hredzak, and V. Agelidis, "Multi-agent sliding mode control for state of charge balancing between battery energy storage systems distributed in a DC microgrid," IEEE Trans. Smart Grid, vol. PP, no. 99, pp. 1-1, 2017.

[26] M. Zeraati, M. E. H. Golshan, and J. Guerrero, "Distributed control of battery energy storage systems for voltage regulation in distribution networks with high PV penetration," IEEE Trans. Smart Grid, vol. PP, no. 99, pp. 1-1, 2016.

[27] N. Mahmud, A. Zahedi, and A. Mahmud, "A cooperative operation of novel PV inverter control scheme and storage energy management system based on ANFIS for voltage regulation of grid-tied PV system," IEEE Trans. Ind. Informat., vol. PP, no. 99, pp. 1-1, 2017.

[28] M. S. Golsorkhi, Q. Shafiee, D. Lu, and J. M. Guerrero, "A distributed control framework for integrated photovoltaic-battery based islanded microgrids," IEEE Trans. Smart Grid, vol. PP, no. 99, pp. 1-1, 2016.

[29] T. L. Vandoorn, B. Meersman, J. D. M. D. Kooning, and L. Vandevelde, "Analogy between conventional grid control and islanded microgrid control based on a global DC-link voltage droop," IEEE Trans. Power Del., vol. 27, no. 3, pp. 1405-1414, July 2012.

[30] — , "Transition from islanded to grid-connected mode of microgrids with voltage-based droop control," IEEE Trans. Power Syst., vol. 28, no. 3, pp. 2545-2553, August 2013.

[31] A. Yazdani and R. Iravani, Voltage-sourced converters in power systems: modeling, control, and applications. John Wiley \& Sons, 2010.

[32] M. S. Rahman, M. J. Hossain, F. H. M. Rafi, and J. Lu, "A multi-purpose interlinking converter control for multiple hybrid AC/DC microgrid operations," in 2016 IEEE Innovative Smart Grid Technologies - Asia (ISGT-Asia), November 2016, pp. 221-226.

[33] B. Singh, P. Jayaprakash, T. R. Somayajulu, and D. P. Kothari, "Reduced rating VSC with a zig-zag transformer for current compensation in a three-phase four-wire distribution system," IEEE Trans. Power Del., vol. 24, no. 1, pp. 249-259, January 2009.

[34] R. Olfati-Saber, J. A. Fax, and R. M. Murray, "Consensus and cooperation in networked multi-agent systems," Proc. IEEE, vol. 95, no. 1, pp. 215-233, January 2007.

[35] R. Olfati-Saber and R. M. Murray, "Consensus problems in networks of agents with switching topology and time-delays," IEEE Trans. Autom. Control, vol. 49, no. 9, pp. 1520-1533, September 2004.

[36] C. Li, E. A. A. Coelho, T. Dragicevic, J. M. Guerrero, and J. C. Vasquez, "Multiagent-based distributed state of charge balancing control for distributed energy storage units in ac microgrids," IEEE Trans. on Ind. Appl., vol. 53, no. 3, pp. 2369-2381, May 2017.

[37] T. K. Refaat, R. M. Daoud, H. H. Amer, and E. A. Makled, "Wifi implementation of wireless networked control systems," in 2010 Seventh International Conference on Networked Sensing Systems (INSS), June 2010, pp. 145-148. 


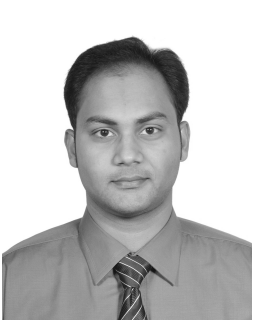

Md Shamiur Rahman (S'14) received the B. Sc. degree in electrical and electronic engineering from Islamic University of Technology (IUT), Bangladesh in 2011. He is currently working toward the Ph.D. degree at the Macquarie University, Sydney, Australia.

Prior to starting Ph.D. studies, he worked as a radio frequency (RF) engineer in Huawei Technologies Bangladesh Limited from 2011 to 2013. Later, from 2013 to 2014 he worked as a core and IP network planning (CNIP) engineer in Robi Axiata Limited. His research interests include microgrid, distributed control techniques, power management, vehicle-to-grid (V2G), energy storage, renewable energy and distributed generation.

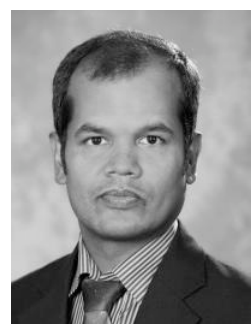

M. Jahangir Hossain (M'10-SM'13) received the B.Sc. and M.Sc. Eng. degrees from Rajshahi University of Engineering and Technology (RUET), Bangladesh, in 2001 and 2005, respectively, and the Ph.D. degree from the University of New South Wales, Australia, all in electrical and electronic engineering.

$\mathrm{He}$ is currently an Associate Professor with the Department of Engineering, Macquarie University. Before joining there, he served as a Senior Lecture and a Lecturer in the Griffith School of Engineering, Griffith University, for five years and as a Research Fellow in the School of Information Technology and Electrical Engineering, University of Queensland, Brisbane, Australia. His research interests include renewable energy integration and stabilization, voltage stability, micro grids and smart grids, robust control, electric vehicles, flexible ac transmission systems devices, and energy storage systems.

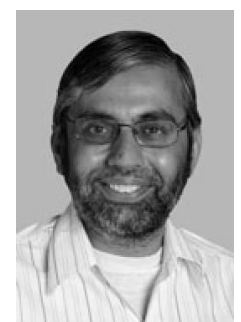

Hemanshu Roy Pota received the B.E. degree from the Sardar Vallabhbhai Regional College of Engineering and Technology, Surat, India, in 1979, the M.E. degree from the Indian Institute of Science, Bangalore, India, in 1981, and the Ph.D. degree from the University of Newcastle, Callaghan, NSW, Australia, in 1985, all in electrical engineering. He is currently an Associate Professor with the University of New South Wales, Australian Defence Force Academy, Canberra, ACT, Australia. He has held visiting appointments with the University of Delaware, Newark, DE, USA; Iowa State University, Ames, IA, USA; Kansas State University, Manhattan, KS, USA; Old Dominion University, Norfolk, VA, USA; the University of California, San Diego, CA, USA; and the Centre for Artificial Intelligence and Robotics, Bangalore, India. His research interests include areas of power system dynamics and control, as well modeling and control of mechanical systems, such as flexible structures, acoustical systems, and UAVs.

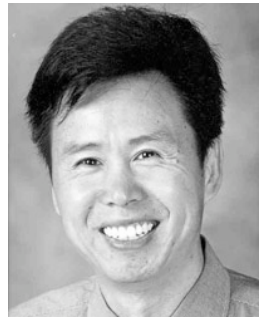

Junwei Lu (M'95-SM'05) received the degree in electrical engineering from Xian Jiaotong University, Xi'an, China, the M.Eng. degree in electronic and computer engineering from the National Toyama University, Toyama, Japan, and the Ph.D. degree in electrical and computer engineering from the National Kanazawa University, Kanazawa, Japan, in 1991.

From 1976 to 1984, he worked with the electrical power industry (now called State Grid) in China, where he was involved in the various national research projects including a $500 \mathrm{kV}$ power system for electrical power industry. In 1985, his academic study and research was in the area of computational electromagnetics at the Laboratory of Electrical Communication Engineering at Toyama University, Toyama Prefecture, Japan. In 1988, he worked on the applied computational electromagnetics and was involved in the development of magnetics devices and electric machines with the Laboratory of Electrical Energy Conversion, Kanazawa University. He joined the School of Microelectronic Engineering, mainly working on power electronics and electric machines, Griffith University, Brisbane, Australia, in 1992, and moved to the Gold Coast campus to establish Electrical and Electronic Engineering as a Foundation Professor in 2011. He has published more than 250 journal and conference papers and three coauthored books in the area of electromagnetic compatibility (EMC) V2G and harmonic balance finite element method, and holds more than 10 international patents related to smart antennas and high frequency transformers. His fields of interest are computational electromagnetics, EMC computer modeling and simulation, radio frequency/microwave devices and circuits, and high-frequency magnetics for power electronics, and renewable energy system. His current research interests include smart transformer and V2G with a built-in statcom inverter and smart hybrid $\mathrm{AC} / \mathrm{DC}$ microgrid. 